\title{
BIOPHYSICS
}

Vol. 7, pp.51-58 (2011)

doi: $10.2142 /$ biophysics.7.51

\section{Functional expression of a two-transmembrane HtrII protein using cell-free synthesis}

\author{
Yuki Sudo ${ }^{1,2}$, Rikou Tanaka ${ }^{3}$, Toshitatsu Kobayashi ${ }^{4}$, Naoki Kamo ${ }^{5}$, Toshiyuki Kohno ${ }^{3}$ \\ and Chojiro Kojima ${ }^{4,6}$ \\ ${ }^{1}$ Division of Biological Science, Graduate School of Science, Nagoya University, Chikusa-ku, Nagoya 464-8602, Japan \\ ${ }^{2}$ PRESTO, Japan Science and Technology Agency (JST), 4-1-8 Honcho Kawaguchi, Saitama 332-0012, Japan \\ ${ }^{3}$ Mitsubishi Kagaku Institute of Life Sciences, Machida, Tokyo 194-0031, Japan \\ ${ }^{4}$ Graduate School of Biological Sciences, Nara Institute of Science and Technology, 8916-5 Takayama, Ikoma, Nara 630-0101, Japan \\ ${ }^{5}$ College of Pharmaceutical Sciences, Matsuyama University, 4-2 Bunkyo-cho, Matsuyama, Ehime 790-8578, Japan \\ ${ }^{6}$ Institute for Protein Research, Osaka University, 3-2 Yamadaoka, Suita, Osaka 565-0871, Japan
}

Received April 8, 2011; accepted May 23, 2011

\begin{abstract}
An approach of cell-free synthesis is presented for the functional expression of transmembrane proteins without the need of refolding. The transmembrane region of the pharaonis halobacterial transducer protein, $p H t r I I$, was translated with various large soluble tags added (thioredoxin, glutathione S-transferase, green fluorescent protein and maltose binding protein). In this system, all fusion $p H t r I I$ were translated in a soluble fraction, presumably, forming giant micelle-like structures. The detergent n-dodecyl- $\beta$-D-maltoside was added for enhancing the solubilization of the hydrophobic region of $p H t r I I$. The activity of the expressed $p H t r I I$, having various tags, was checked using a pull-down assay, using the fact that $p H$ trII forms a signaling complex with pharaonis phoborhodopsin ( $p \mathrm{pR})$ in the membrane, as also in the presence of a detergent. All tagged $p H$ trII
\end{abstract}

Abbreviations: $p$ HtrII, pharaonis halobacterial transducer II; $p \mathrm{pR}$, pharaonis phoborhodopsin; GST, glutathione S-transferase; MBP, maltose binding protein; Trx, thioredoxin; GFP, green fluorescent protein; DDM, n-dodecyl- $\beta$-D-maltoside; Y199A, Y199A $p$ pR mutant in which Tyr-199 is substituted by Ala

* This work was supported by grants from the Japanese Ministry of Education, Culture, Sports, Science, and Technology, Japan.

Corresponding author: Yuki Sudo, Division of Biological Science, Graduate School of Science, Nagoya University, Chikusa-ku, Nagoya, 464-8602, Japan. e-mail: z47867a@cc.nagoya-u.ac.jp; Chojiro Kojima, Institute for Protein Research, Osaka University, 3-2 Yamadaoka, Suita, Osaka 565-0871, Japan. e-mail: kojima@protein.osaka-u.ac.jp showed a binding activity with $p p R$. Interestingly, the binding activity with $p p R$ was positively correlated with the molecular weight of the soluble tags. Thus, larger soluble tags lead to higher binding activities. We could show, that our approach is beneficial for the preparation of active membrane proteins, and is also potentially applicable for larger membrane proteins, such as 7transmembrane proteins.

Key words: membrane protein, cell-free protein synthesis, protein-protein interaction, sensory rhodopsin

About $25-30 \%$ of proteins are embedded in membranes. Thus, one of the most popular areas of research in biology is the functional analysis of these membrane proteins ${ }^{1}$. However, these proteins are insoluble in aqueous solutions, and difficult to overexpress and purify. Three strategies are currently being used for the protein expression: chemical synthesis, in vivo expression, and cell-free protein synthesis. However, the former two methods have certain limitations: chemical synthesis is not feasible for the synthesis of long peptides because of its low yield, and in vivo expression can produce only those proteins that do not affect the physiology of the host cell ${ }^{2,3}$. Furthermore, even if they are expressed, it requires many steps for the protein purification: membrane proteins expressed in cells have to be solubilized by a detergent, purified by an affinity column, and reconstituted into phospholipids. Cell-free translation 
a)

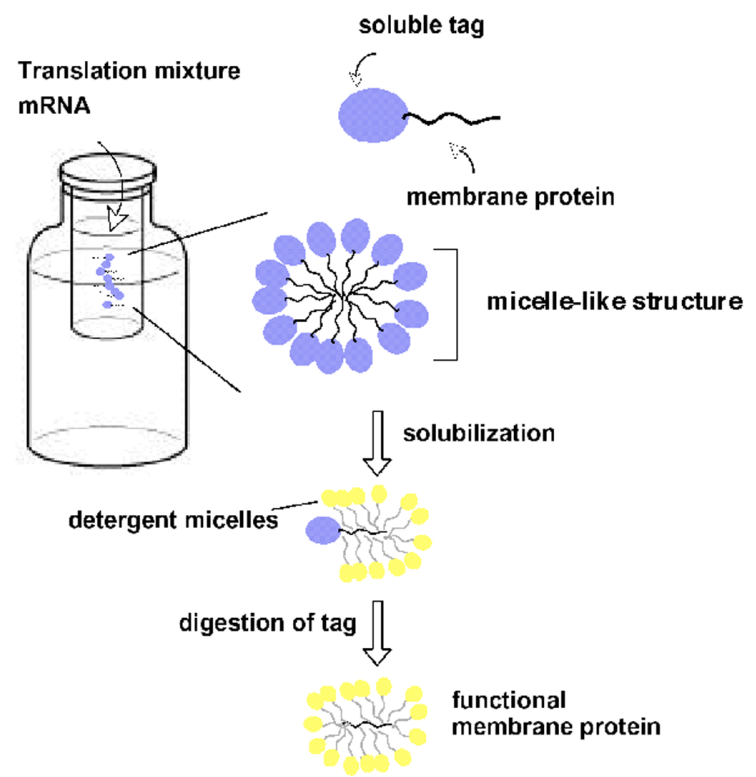

b)

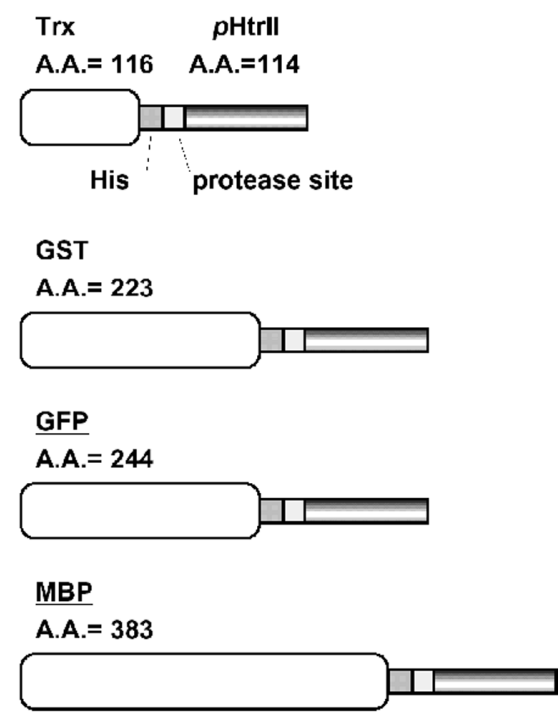

Figure 1 Schematic drawing of the used approach. (a) Translation products are soluble in the reaction solution forming giant micelles. Then, the detergent DDM was added to the solution for the solubilization of the hydrophobic regions. Finally, the tag was digested by a protease, and the functional membrane protein was prepared. (b) $p$ HtrII ${ }^{1-114}$ with large soluble tags expressed in the cell-free system. The proteins have a histidine tag and a protease site which can be used for the pull-down assay or the tag digestion, respectively.

systems, in contrast, can synthesize proteins with high speed and accuracy, approaching in vivo rates $^{4}$, and they can express proteins that would interfere with the cell physiology $\mathrm{y}^{5,6}$.

In previous reports, some membrane proteins have been successfully expressed in cell-free system ${ }^{7,8}$. However, because proteins tend to aggregate in cell-free systems, a refolding process of the proteins is, in general, required. Therefore, these proteins have been expressed in the presence of a detergent to solubilize the protein, or in the presence of lipids to reconstitute the protein ${ }^{9-11}$. However, the yield of such expressed functional proteins was very low. Therefore, a new strategy is required for the expression of membrane proteins by using cell-free protein synthesis.

In the present study we developed a strategy for the membrane protein expression by using a cell-free protein synthesis system (Fig. 1a). A wheat germ cell-free system was chosen, because in it the translation reaction can proceed for longer than $60 \mathrm{hrs}$, and active proteins are yielded in milligram quantities per milliliter reaction volume. The membrane region of the pharaonis halobacterial transducer protein, $p$ HtrII, was translated with various large soluble tags (thioredoxin, glutathione S-transferase, green fluorescent protein or maltose binding protein) (Fig. 1b). pHtrII has two transmembrane helices, and belongs to the family of two-transmembrane helical methyl-accepting chemotaxis proteins (MCPs) $)^{12-14}$. It forms a signaling complex with pharaonis phoborhodopsin ( $p \mathrm{pR}$, also called pharaonis sensory rhodopsin II, $p$ sRII) in the halobacterial membrane ${ }^{15}$.
This complex transmits a light signal to the sensory system which is called the two-component system in the cytoplasm $^{13} \cdot p$ HtrII activates phosphorylation cascades which modulate flagellar motors ${ }^{14}$. By using these signaling systems, Natronomonas pharaonis cells avoid harmful nearUV light, a behaviour called negative phototaxis. It is known that both, $p \mathrm{HtrII}$ and $p \mathrm{pR}$, are stable in membranes as well as in detergent micelles ${ }^{16-18}$, and both have been well characterized over the past few years using various methods ${ }^{15,19}$. Therefore, we chose to use $p$ HtrII as a model for membrane proteins.

\section{MATERIALS AND METHODS}

\section{Protein synthesis}

Commercially available tag genes, Trx, GST, GFP and MBP, were used here. They are cloned into a plasmid vector and all constructed plasmids were analyzed using an automated sequencer to confirm the expected nucleotide sequences. In the presence of $16 \mathrm{mM} \mathrm{Mg}^{2+}$, mRNAs of various soluble tagged $p$ HtrIIs were synthesized with a SP6 RNA polymerase, using the plasmids as templates. These synthesized mRNAs were precipitated with ethanol, dissolved in a dialysis buffer, and then mixed with the wheat germ extract for protein synthesis. The purification of the wheat embryos, and the preparation of the cell-free extract were performed as described previously ${ }^{20}$. Fusion $p H$ trII, displayed in Figure 1b, were expressed as a soluble fraction (Fig. 2), to which $0.1 \%$ DDM was added for 1 hour for solu- 


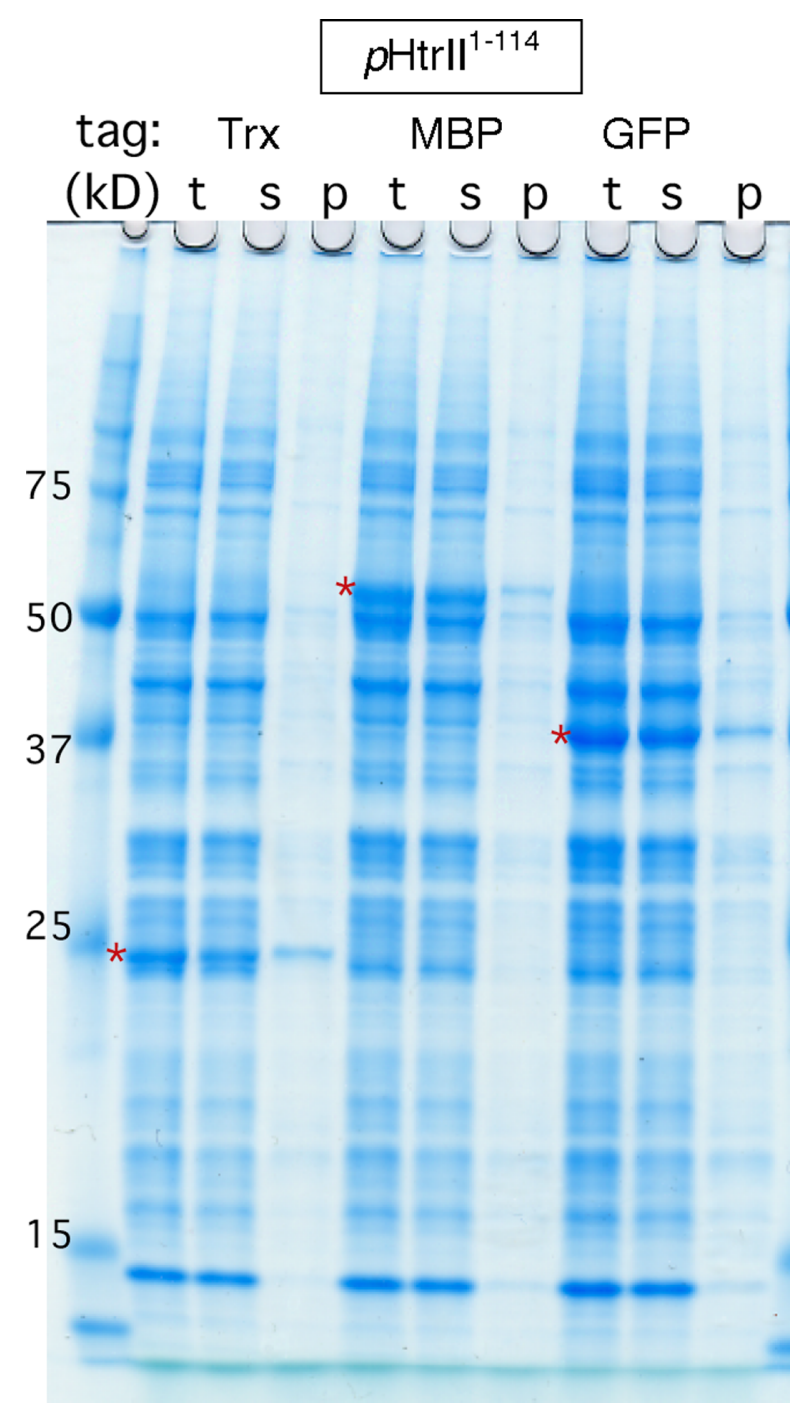

Figure 2 SDS-PAGE analysis of cell-free protein synthesis of tagged $p \mathrm{HtrII}$. T, $\mathrm{S}$ and $\mathrm{P}$ represent total translational product, soluble fraction and pellet after low speed centrifugation, respectively. The bands marked by a star in the gel represent the tagged $p \mathrm{HtrII}^{1-114}$ obtained by cell-free translation.

bilization at room temperature under gentle stirring.

\section{Analysis of the activity of the soluble tagged pHtrII}

An in vitro pull-down assay was performed using a NiNTA column, essentially, as previously described ${ }^{21-23} \cdot p \mathrm{pR}$ and $p \mathrm{HtrII}^{1-159} \mathrm{His}$ were expressed in Escherichia coli ${ }^{21,24}$. Here, His denotes a tag with six histidine residues attached at the C-terminus of the protein. It was previously shown that truncated $p \mathrm{HtrII}$, expressed from position 1 to position $159\left(p \mathrm{HtrII}^{1-159}\right)$, is sufficient to permit the interaction with $p p \mathrm{R}^{21,25,26}$. Furthermore, Engelhard and coworkers reported that also $p \mathrm{HtrII}$, expressed from position 1 to position 114, had a full binding activity with $p \mathrm{pR}^{26}$, and that $p \mathrm{HtrII}^{1-114}$ is a more hydrophobic protein than $p \mathrm{HtrII}^{1-159}$. Therefore, we used mainly $p \mathrm{HtrII}^{1-114}$ in this study as a model for mem- brane proteins. The preparation of crude membranes, and the purification of proteins were performed as previously described $^{21,24}$. The sample medium was exchanged by Amicon Ultra (Millipore, Bedford, MA) filtration, and the samples were suspended in buffer $\mathrm{S}(300 \mathrm{mM} \mathrm{NaCl}, 50 \mathrm{mM}$ MES, $5 \mathrm{mM}$ Imidazole, pH6.5) containing 0.1\% DDM. Purified untagged $p \mathrm{pR}(0.8 \mathrm{mM})$ and histidine tagged $p \mathrm{HtrII}$ $(0.08 \mathrm{mM})$, with various soluble tags, were mixed in the molar ratio of $1: 10$ in buffer $\mathrm{S}$ containing $0.1 \% \mathrm{DDM}$ for 1 hour at room temperature under gentle stirring. The Ni-NTA resin was washed extensively with buffer W (0.1\% DDM, $300 \mathrm{mM} \mathrm{NaCl}, 50 \mathrm{mM}$ MES, $50 \mathrm{mM}$ Imidazole, $\mathrm{pH} 6.5)$ to remove non-specifically bound proteins. The $p \mathrm{pR} / p \mathrm{HtrIIs}$ complexes were eluted with buffer E (0.1\% DDM, $300 \mathrm{mM}$ $\mathrm{NaCl}, 50 \mathrm{mM}$ Tris- $\mathrm{HCl}, 150 \mathrm{mM}$ Imidazole, $\mathrm{pH}$ 7.0), and the absorbance at $498 \mathrm{~nm}$ was measured (V-560 spectrophotometer, Japan, Spectroscopic, Tokyo, Japan). As $p$ pR maximally absorbs light at $498 \mathrm{~nm}^{27}$, which is not affected by the binding of $p \mathrm{HtrII}^{21,28}$, the concentration of $p \mathrm{pR}$ could be easily detected by the color and the absorbance.

\section{Interaction between the un-tagged pHtrII and ppR}

MBP-pHtrII $(0.1 \mathrm{mM})$ was incubated with PreScission protease $(5 \mu \mathrm{g} / \mathrm{mL})$ for $5 \mathrm{hrs}$ at $10^{\circ} \mathrm{C}$. The reaction was stopped by the addition of a protease inhibitor (APMSF), and the digested fragments were separated by Ni-affinity chromatography. Purified histidine tagged $p \mathrm{pR}(0.01 \mathrm{mM})$ and $p \mathrm{HtrII}$, derived from MBP- $p \mathrm{HtrII}(0.1 \mathrm{mM})$, or $p \mathrm{HtrII}^{1-159}$ $(0.1 \mathrm{mM})$ were mixed in buffer $\mathrm{S}$, the Ni-NTA resin was washed extensively with buffer $\mathrm{W}$, and the $p \mathrm{pR} / p \mathrm{HtrII}$ complexes were eluted with buffer $E$. These proteins were separated by $12 \%$ SDS-PAGE.

\section{Activity of pHtrII solubilized by various detergents}

We used $0.1 \%$ DDM, $1 \%$ n-octyl- $\beta$-D-glucoside (OG), $1 \%$ n-octyl-thio- $\beta$-D-glucoside (OTG), $20 \mathrm{mM}$ cholic acid, $15 \mathrm{mM}$ SDS or $12 \mathrm{mM}$ CHAPS as a detergent for the solubilization of $p$ HtrII. Translated MBP- $p \mathrm{HtrII}$ was solubilized by DDM, OG, OTG, cholic acid, SDS or CHAPS for 1 hour at room temperature under gentle stirring. Then, the suspending medium was completely exchanged by dialysis against buffer $\mathrm{S}$ containing $0.1 \%$ DDM for one week, because $p \mathrm{pR}$ loses its activity in detergent solutions, except for those containing DDM, OG or OTG.

\section{Expression of large soluble tagged pHtrII in cells and its interaction with $\mathbf{p p R}$}

GST- $p$ HtrII was expressed in E. coli BL21 (DE3) star by induction initialized by the the addition of $1 \mathrm{mM}$ IPTG. The expressed proteins were suspended in buffer A $(5 \mathrm{mM}$ $\mathrm{MgCl}_{2}, 50 \mathrm{mM}$ Tris-Cl, $\mathrm{pH} 8.0$ ) containing $1 \mathrm{mM}$ dithiothreitol (DTT) and $8 \mathrm{M}$ urea, and refolded by the addition of 50 -fold diluted buffer A. A detergent (0.1\% DDM) was added after this rapid dilution at $15^{\circ} \mathrm{C}$ for the solubilization of the membrane helical region of GST- $p$ HtrII. The Y199A 
mutant of the $p \mathrm{pR}$ gene was prepared by PCR using the QuickChange method. The prepration of crude membranes and the purification of proteins were performed as previously described ${ }^{25}$. The sample medium was exchanged by Amicon Ultra filtration, and the samples were suspended in buffer A containing 0.1\% DDM. Purified $p \mathrm{pR}(0.8 \mathrm{mM})$ and GST- $p$ HtrII $(0.08 \mathrm{mM})$ were mixed in buffer A containing $0.1 \% \mathrm{DDM}$, and incubated for 1 hour at room temperature under gentle stirring. The samples were applied to a glutathione sepharose 4B resin, filled into a chromatography column, and extensively washed with buffer A containing $0.1 \%$ DDM to remove non-specifically bound proteins. The $p$ pR/GST- $p$ HtrII complex was eluted with buffer GE (0.1\% DDM, $5 \mathrm{mM} \mathrm{MgCl}$, $50 \mathrm{mM}$ Tris-Cl, pH 8.0, $30 \mathrm{mM}$ glutathione (reduced form)). The absorbance of the elution fractions was measured at $498 \mathrm{~nm}$.

\section{RESULTS}

\section{Translational products}

In our system, translation products were mainly soluble in the reaction solution (Fig. 2). Furthermore, for example, GST- $p$ HtrII was even soluble in the absence of a detergent, and formed large complexes of a size of about $10 \mathrm{Mda}$, estimated by dynamic light scattering. The crude cell-free translation mixture without detergent was used in these experiments (i.e., soluble fraction in Fig. 2). This could be explained by the formation of hydrophobic and hydrophilic clusters by the hydrophobic (membrane) and hydrophilic (tag) regions of the proteins, respectively, leading to structures such as giant micelles or lipid bilayers (Fig. 1). However, because of the instability of $p \mathrm{pR}$ in the absence of the detergent, $0.1 \%$ DDM, a comparably mild detergent, was added to all these soluble tagged $p H$ trII, used in the experiments.

\section{Activity of soluble tagged pHtrII}

In an effort to determine if the soluble tagged $p \mathrm{HtrII}$ is functional, we performed an in vitro pull-down assay (see Materials and Methods, and refs 21-23). ppR was adsorbed onto a Ni-NTA resin containing immobilized histidine tagged fusion $p H$ trII proteins. Figure 3 shows the adsorbed fraction of $p \mathrm{pR}$ in the absence (lane 1) and presence of Trx$p$ HtrII (lane 2), GST- $p$ HtrII (lane 3), GFP- $p$ HtrII (lane 4), MBP- $p \mathrm{HtrII}$ (lane 5) and $p \mathrm{HtrII}^{1-159}$ (lane 6). $p \mathrm{HtrII}^{1-159}$ was expressed in E. coli cells, purified by the column chromatography and used as a positive control. Because $p \mathrm{pR}$ maximally absorbs light at $498 \mathrm{~nm}$, the concentration of $p \mathrm{pR}$ can easily be determined by the color and the absorbance (Fig. 3). Specifically adsorbed $p p R$ was detected in the presence of all fusion $p \mathrm{HtrII}$, indicating that all tagged $p \mathrm{HtrII}$ have a certain binding activity to $p \mathrm{pR}$. As already mentioned, in these experiments, purified untagged $p \mathrm{pR}(0.8 \mathrm{mM})$ and all histidine tagged $p$ HtrII $(0.08 \mathrm{mM})$ were mixed in the molar ratio of $1: 10$. Therefore, the bound fraction of $p \mathrm{pR}$ to $p \mathrm{HtrII}$

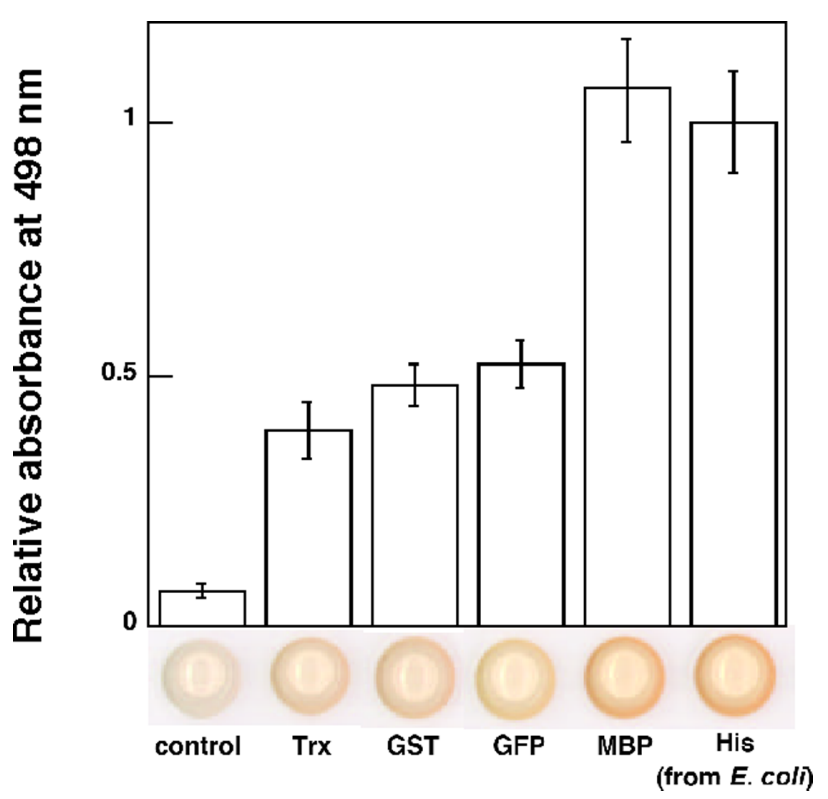

Figure 3 In vitro pull-down assay using a Ni-NTA resin. $p \mathrm{pR}$ was applied to the column without $p$ HtrII (control) and with Trx-tagged $p$ HtrII (Trx), GST-tagged $p$ HtrII (GST), GFP-tagged $p$ HtrII (GFP), MBP-tagged $p \mathrm{HtrII}(\mathrm{MBP})$ and $p \mathrm{HtrII}{ }^{1-159} \mathrm{His}$ (His(from E. coli), as a positive control). After the column was extensively washed with buffer W (for details, see Materials and Methods) to remove nonspecifically bound proteins, bound proteins were eluted with buffer $\mathrm{E}$ (see Materials and Methods). The eluted material was collected, and the UV-vis spectrum of $p \mathrm{pR}(\lambda \max =498)$ was then measured. The circles display the color and absorbance of $p \mathrm{pR}$ bound to $p \mathrm{HtrII}$ for each column. All fusion $p H$ trII represent functional proteins because the fractions of $p \mathrm{pR}$ bound to $p \mathrm{HtrII}$ are much higher than that of the control.

represents the activity related to the folded fraction of soluble tagged $p$ HtrII.

It has been reported that $p$ HtrII forms a complex with $p$ pR with a 2:2 stoichiometry under a number of conditions, such as in detergents, in crystals and lipids ${ }^{21,22,26,29-31}$. Therefore, $p \mathrm{HtrII}$, expressed in E. coli, could be used as a control having the full binding activity to $p \mathrm{pR}$ with folded structures. As can be seen in figure 2, three of the soluble tagged $p$ HtrII (Trx, GST and GFP) had a binding activity of only about $50 \%$ that of $p \mathrm{HtrII}$, while the forth tagged form (MBP) acts similar to the wildtype. This suggests that an unfolded fraction exists in these three tagged $p$ HtrII (Trx, GST and GFP), although the translated proteins were mainly soluble in solution (Fig. 2). From these analyses, we could conclude that all four soluble tagged $p H$ trII, expressed in the cell-free system, were functional, although the fraction of functional proteins depended on the tag used. As already mentioned, the MBP-tagged $p H t r I I$ has the highest activity. This is interesting given that MBP is the largest tag among the soluble tags used showing the highest activity. Both values, binding activity and the molecular weight of the tags seem to be positively correlated (Fig. 3). As the soluble tag surrounds the hydrophobic region of $p \mathrm{HtrII}$, the soluble 
(1)

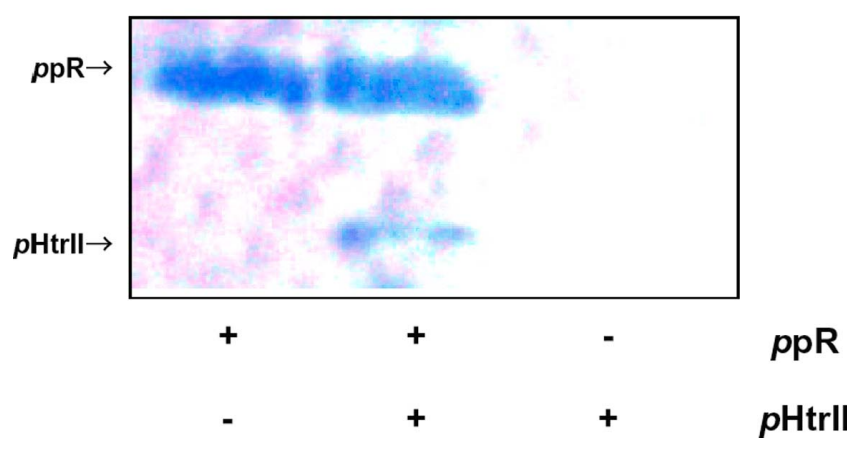

Figure 4 In vitro pull-down assay using a Ni-NTA resin. $p$ pR was applied to the column without $p \mathrm{HtrII}$ (lane 1) and with MBP digested $p \mathrm{HtrII}$ (lane 2) and with MBP digested $p \mathrm{HtrII}$ in the absence of $p \mathrm{pR}$ (lane 3). After the column was extensively washed with buffer W (for details, see Materials and Methods) to remove nonspecifically bound proteins, bound proteins were eluted with buffer E (see Materials and Methods). The eluted material was collected, and the SDS-PAGE was performed. $p$ HtrII was only detected in the presence of both, $p \mathrm{pR}$ and $p \mathrm{HtrII}$, indicating that MBP digested $p \mathrm{HtrII}$ is functional.

tag may support the exact folding of $p H$ trII, and prevent the aggregation of the hydrophobic regions of $p \mathrm{HtrII}$, which is more effective when the size of the tag is large.

\section{Activity of MBP-digested pHtrII}

For the preparation of un-tagged membrane proteins, it is necessary to digest the fusion soluble tag. Then, the binding activity to $p \mathrm{pR}$ was checked using a pull-down assay. For this, $p$ HtrII was adsorbed onto a Ni-NTA resin containing immobilized histidine tagged fusion $p \mathrm{pR}$. Figure 4 shows the adsorbed fraction of $p \mathrm{HtrII}$, in the presence of histidine tagged $p \mathrm{pR}$ without $p \mathrm{HtrII}$ (lane 1), in the presence of both, $p \mathrm{pR}$ and MBP-digested $p \mathrm{HtrII}$ (lane 2), and for MBPdigested $p \mathrm{HtrII}$ in the absence of $p \mathrm{pR}$ (lane 3 ). As expected, specifically adsorbed $p$ HtrII was only detected in the presence of both, $p \mathrm{pR}$ and $p \mathrm{HtrII}$. The results show that also tagdigested $p$ HtrII is functional.

\section{Detergent dependency on the activity of MBP tagged pHtrII}

In this report, we used DDM as a detergent for the solubilization of $p$ HtrII. However, the kind of detergent used might have an influence on the activity of the tagged protein, and therefore, we examined the detergent dependency on the activity of the MBP tagged $p H$ trII (Fig. 5). $p H$ trII solubilized in non-ionized detergents (DDM, OG and OTG) had a much higher activity than in ionic (cholic acid and SDS) and amphoteric (CHAPS) detergents. Thus, in a cellfree protein synthesis system, non-ionized detergents might be the best choice for solubilization of tags, as it is at least in the present system. These results are consistent with previous results showing that $p \mathrm{pR}$ and $p \mathrm{HtrII}$ are not stable in the presence of cholic acid, SDS and CHAPS (ref 32 and unpublished data).

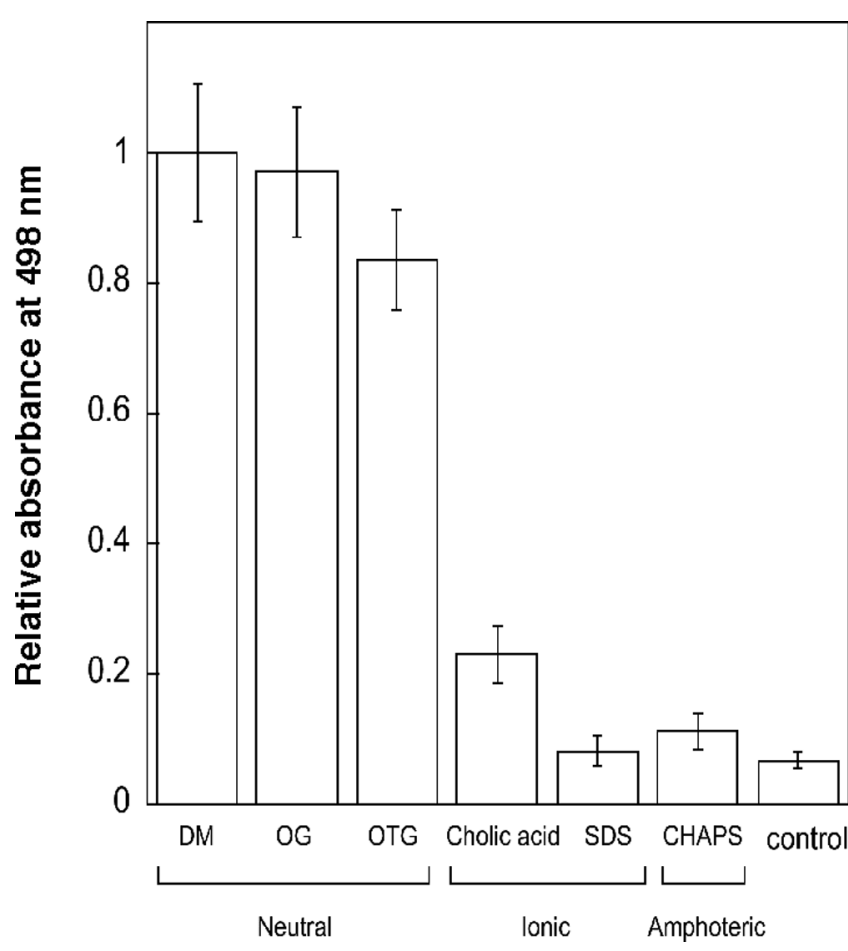

Figure 5 Effect of he type of detergent on the activity of MBP$p$ HtrII. MBP- $p$ HtrII was solubilized by DDM, OG, OTG, Cholic acid, SDS and CHAPS, and exchanged completely by dialysis against buffer S (for details, see Materials and Methods) containing $0.1 \%$ DDM. $p \mathrm{pR}$ was applied to the column without $p$ HtrII (control) and with MBP-tagged $p$ HtrII. After the column was extensively washed with buffer W (for details, see Materials and Methods) to remove nonspecifically bound proteins, bound proteins were eluted with buffer E (see Materials and Methods). The eluted material was collected, and then the UV-vis spectrum of $p \mathrm{pR}(\lambda \max =498)$ was measured.

\section{DISCUSSION}

In the past, membrane proteins without soluble tags have been successfully expressed in cell-free system, however, the protein formed aggregates, and thus, a refolding processes were required ${ }^{7,8}$. In this report, we succeeded in designing an approach of cell-free protein expression in which refolding is not needed. The translation products in our system were soluble in the reaction solution forming micelle-like or lipid bilayer-like structures. Among others, the detergent DDM was added into the solution for the solubilization of the hydrophobic regions. Using various non-ionized detergents, we could obtain functional $p H$ trII (Fig. 5). It is well known that the electro-neutral detergents, DDM, OG and OTG are mild agents for the solubilization of native cell membranes because the lengths of their alkyl chains are all similar, and moreover, almost identical to the hydrophobic chains of lipids ${ }^{33,34}$. In contrast, ionic and amphoteric detergents are not mild agents, and lead to a comparably low activity of the proteins. Therefore, nonionized detergent may be the best choice for the maintenance of the protein structure. However, the detailed struc- 
ture of the soluble tagged $p H$ trII in solution in the absence of detergents is still not known, and thus, further studies are necessary. Nonetheless, in all cases, our membrane protein synthesis system did not lead to irreversibly aggregated states of the proteins, and thus, it was not necessary to refold the proteins. Moreover, MBP-digested $p$ HtrII could be shown to be as functional as $p \mathrm{HtrII}$ obtained by an in vitro expression system (Fig. 4). Therefore, the highthroughput functional expression of $p$ HtrII was easily achievable.

In this study, we demonstrated that a larger soluble tagged $p \mathrm{HtrII}$ has a higher $p$ HtrII activity (see Figs. 1 and 3 ). It can be expected that membrane protein which have even larger transmembrane (hydrophobic) regions, as e.g., seventransmembrane helix proteins, as is the G-protein coupled receptor (GPCR), can be only expressed in the cell-free protein synthesis by adding even more hydrophilic tags. While, in the case of $p H$ trII, whose molecular weight is about $10 \mathrm{kDa}$, a $40 \mathrm{kDa}$ soluble tag was sufficient for the preparation of a fully active $p$ HtrII (Fig. 3), for seven-transmembrane proteins with much larger hydrophobic region whose molecular weight is about $20 \mathrm{kDa}$, it will be necessary, for ensuring the functional expression in the cell-free protein synthesis, to add the soluble tags whose molecular weight is about $80 \mathrm{kDa}$. Such a $80 \mathrm{kDa}$ soluble tag could be, for example, possible prepared by combining a MBP tag with four Trx or two GFP tags.

It can be assumed that the soluble tags may enhance the protein expression level not only in the cell free protein synthesis but also in the cell protein synthesis. To verify this, GST-tagged $p H$ trII, including the transmembrane region of $p$ HtrII, was expressed in E. coli. The obtained expression levels and yields were comparably high $(100 \mathrm{mg} / \mathrm{L}$ culture), however, because of the high protein concentration in the cells, GST-tagged $p$ HtrII forms aggregation (inclusion body), therefore a refolding process was required. In order to determine if the obtained $p H$ trII is functional, a GST pull-down assay was performed (see Materials and Methods, and refs 21-23). Figure 6 shows the relative Optical Density (OD) at $498 \mathrm{~nm}$ from the adsorbed fractions of $p \mathrm{pR}$ alone (control), the mixture containing a $p \mathrm{pR}$ Y199A mutant and GST$p$ HtrII (Y199A), and the mixture containing wild type $p \mathrm{pR}$ and GST- $p$ HtrII (W.T.). The mutant was chosen, because Tyr199 of $p \mathrm{pR}$ is one of the most important residues for the interaction with $p \mathrm{HtrII}^{17,26,29}$, and therefore a lower binding affinity could be expected compared to WT $p \mathrm{pR}$. This could be confirmed as the fraction of the binding of the Y199A $p \mathrm{pR}$ mutant to $p \mathrm{HtrII}$ was lower than that of W.T. $p \mathrm{pR}$ (Fig. $6)$. The results suggest that GST- $p$ HtrII can interact with $p \mathrm{pR}$, and that this pull-down system functions normally. Furthermore, in this system it is comparably easy to prepare large amounts of functional $p H$ trII. Also, the in vitro pulldown assay used here is a simpler and easier method compared to the photo-chemical ${ }^{21,22,25,29}$ or calorimetric methods $^{23,26}$ used so far for analyzing the interaction

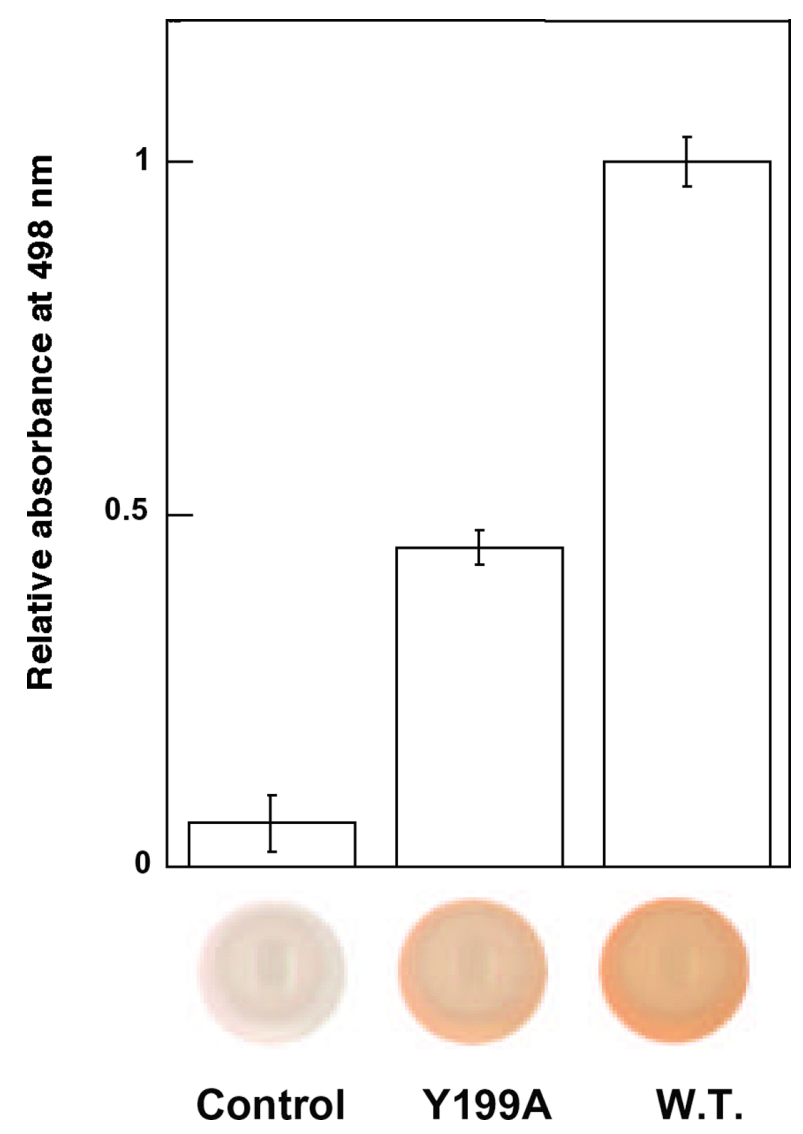

Figure 6 Binding activity of GST- $p$ HtrII expressed in a cell to $p \mathrm{pR}$. The relative binding activities of $p \mathrm{HtrII}$ to $p \mathrm{pR}$ without $p \mathrm{HtrII}$ (left), the mixture containing the $p \mathrm{pR}$ Y199A mutant and $p \mathrm{HtrII}$ (center) and the mixture containing $p \mathrm{pR}$ and $p \mathrm{HtrII}$ (right) are shown. The circle display the color of the sample obtained after elution with buffer GE (for details, see Materials and Methods). The color originates from $p$ pR which absorbs at $\sim 500 \mathrm{~nm}$. About $100 \mathrm{mg}$ functional protein was produced in a 1 liter culture.

between $p \mathrm{HtrII}$ and $p \mathrm{pR}$. Therefore, it can be concluded that the use of large soluble tags can enhance the membrane protein expression level in both, the cell and cell-free systems.

In summary, it is, in general, difficult to prepare membrane proteins using either chemical synthesis or in vivo expression. In this paper, we have succeeded in developing a membrane protein expression system using a cell-free protein synthesis with solubility enhancing tags. All tagged $p$ HtrII were translated in a soluble fraction, in which the hydrophobic regions of $p$ HtrII and the hydrophilic tags might form giant micelle-like structures (Fig. 1). All tagged $p$ HtrII had a binding activity with $p \mathrm{pR}$. Interestingly, by increasing the molecular weight of the tags a larger active fraction of the protein could be yielded. These data suggest the possibility to express membrane proteins with a larger hydrophobic region, such as 3 7 transmembrane proteins, using larger soluble tags. 


\section{Acknowledgment}

We thank Drs Kazumi Shimono (Matsuyama University) and Masayuki Iwamoto (Fukui university) for diverse useful discussions. We also thank Dr. Louisa Reissig for critical reading of our manuscript.

\section{REFERENCES}

1. Kyogoku, Y., Fujiyoshi, Y., Shimada, I., Nakamura, H., Tsukihara, T., Akutsu, H., Odahara, T., Okada, T. \& Nomura, N. Structural genomics of membrane proteins. Acc. Chem. Res. 36, 199-206 (2003).

2. Goff, S. A. \& Goldberg, A.L. An increased content of protease $\mathrm{La}$, the lon gene product, increases protein degradation and blocks growth in Escherichia coli. J. Biol. Chem. 262, 4508-4515 (1987).

3. Henrich, B., Lubitz, W. \& Plapp, R. Lysis of Escherichia coli by induction of cloned phi X174 genes. Mol. Gen. Genet. 185, 493-497 (1982).

4. Kurland, C. G. Translational accuracy in vitro, Cell 28, 201202 (1982).

5. Jermutus, L., Ryabova, L.A. \& Plückthun, A. Recent advances in producing and selecting functional proteins by using cell-free translation. Curr. Opin. Biotechnol. 9, 534548 (1998).

6. Endo, Y. \& Sawasaki, T. High-throughput, genome-scale protein production method based on the wheat germ cell-free expression system. Biotechnol. Adv. 21, 695-713 (2003).

7. Sonar, S., Lee, C.P., Coleman, M., Patel, N., Liu, X., Marti, T., Khorana, H. G., Rajbhandary, U.L. \& Rothschild, K. J. Site-directed isotope labelling and FTIR spectroscopy of bacteriorhodopsin. Nat. Struct. Biol. 1, 512-517 (1994).

8. Bergo, V., Mamaev, S., Olejnik, J. \& Rothschild, K. J. Methionine changes in bacteriorhodopsin detected by FTIR and cell-free selenomethionine substitution. Biophys. J. 84, 960-966 (2003).

9. Klammt, C., Löhr, F., Schäfer, B., Haase, W., Dötsch, V., Rüterjans, H., Glaubitz, C. \& Bernhard, F. High level cell-free expression and specific labeling of integral membrane proteins. Eur. J. Biochem. 271, 568-580 (2004).

10. Ishihara, G., Goto, M., Saeki, M., Ito, K., Hori, T., Kigawa, T., Shirouzu, M. \& Yokoyama S. Expression of G protein coupled receptors in a cell-free translational system using detergents and thioredoxin-fusion vectors. Protein Expr. Purif. 41, 27-37 (2005).

11. Shimono, K., Goto, M., Kikukawa, T., Miyauchi, S., Shirouzu, M., Kamo, N. \& Yokoyama, S. Production of functional bacteriorhodopsin by an Escherichia coli cell-free protein synthesis system supplemented with steroid detergent and lipid. Protein Sci. 18, 2160-2171 (2009).

12. Rudolph, J., Nordmann, B., Storch, K. F., Gruenberg, H., Rodewald, K. \& Oesterhelt, D. A family of halobacterial transducer proteins. FEMS Microbiol. Lett. 139, 161-168 (1996).

13. Falke, J.J., Bass, R.B., Butler, S.L., Chervitz, S.A. \& Danielson, M.A. The two-component signaling pathway of bacterial chemotaxis: a molecular view of signal transduction by receptors, kinases, and adaptation enzymes. Annu. Rev. Cell Dev. Biol. 13, 457-512 (1997).

14. Hoff, W.D., Jung, K. H. \& Spudich, J.L. Molecular mechanism of photosignaling by archaeal sensory rhodopsins. Annu. Rev. Biophys. Biomol. Struct. 26, 223-258 (1997).

15. Sudo, Y., Kandori, H. \& Kamo, N. Molecular mechanism of protein-protein interaction of pharaonis phoborhodopsin/transducer and photo-signal transfer reaction by the complex. Recent Res. Devel. Biophys. 3, 1-16 (2004).

16. Ikeura, Y., Shimono, K., Iwamoto, M., Sudo, Y. \& Kamo, N. Arg-72 of pharaonis phoborhodopsin (sensory rhodopsin II) is important for the maintenance of the protein structure in the solubilized state. Photochem. Photobiol. 77, 96-100 (2003).

17. Sudo, Y., Yamabi, M., Iwamoto, M., Shimono, K. \& Kamo, N. Interaction of Natronobacterium pharaonis phoborhodop$\sin$ (sensory rhodopsin II) with its cognate transducer probed by increase in the thermal stability. Photochem. Photobiol. 78, 511-516 (2003).

18. Sudo, Y., Furutani, Y., Shimono, K., Kamo, N. \& Kandori, H. Hydrogen bonding alteration of Thr-204 in the complex between pharaonis phoborhodopsin and its transducer protein. Biochemistry 42, 14166-14172 (2003).

19. Klare, J. P., Gordeliy, V. I., Labahn, J., Büldt, G., Steinhoff, H.J. \& Engelhard, M. The archaeal sensory rhodopsin II/transducer complex: a model for transmembrane signal transfer. FEBS Lett. 564, 219-224 (2004).

20. Morita, E.H., Shimizu, M., Ogasawara, T., Endo, Y., Tanaka, R. \& Kohno, T. A novel way of amino acid-specific assignment in (1)H-(15)N HSQC spectra with a wheat germ cellfree protein synthesis system. J. Biomol, NMR 30, 37-45 (2004).

21. Sudo, Y., Iwamoto, M., Shimono, K. \& Kamo, N. Pharaonis phoborhodopsin binds to its cognate truncated transducer even in the presence of a detergent with a 1:1 stoichiometry. Photochem. Photobiol. 74, 489-494 (2001).

22. Sudo, Y., Iwamoto, M., Shimono, K. \& Kamo, N. Association between a photo-intermediate of a M-lacking mutant D75N of pharaonis phoborhodopsin and its cognate transducer. J. Photochem. Photobiol. B 67, 171-176 (2002).

23. Sudo, Y., Okuda, H., Yamabi, M., Fukuzaki, Y., Mishima, M., Kamo, N. \& Kojima, C. Linker region of a halobacterial transducer protein interacts directly with its sensor retinal protein. Biochemistry 44, 6144-6152 (2005).

24. Kandori, H., Shimono, K., Sudo, Y., Iwamoto, M., Shichida, Y. \& Kamo, N. Structural changes of pharaonis phoborhodopsin upon photoisomerization of the retinal chromophore: Infrared spectral comparison with bacteriorhodopsin. Biochemistry 40, 9238-9246. (2001).

25. Sudo, Y., Iwamoto, M., Shimono, K. \& Kamo, N. Role of charged residues of pharaonis phoborhodopsin (seonsory rhodopsin II) in its interaction with the transducer protein. Biochemistry 43, 13748-13754 (2004).

26. Hippler-Mreyen, S., Klare, J.P., Wegener, A. A., Seidel, R., Herrmann, C., Schmies, G., Nagel, G., Bamberg, E. \& Engelhard, M. Probing the sensory rhodopsin II binding domain of its cognate transducer by calorimetry and electrophysiology. J. Mol. Biol. 330, 1203-1213 (2003).

27. Shimono, K., Iwamoto, M., Sumi, M. \& Kamo, N. Functional expression of pharaonis phoborhodopsin in Escherichia coli. FEBS Lett. 420, 54-56 (1997).

28. Sudo, Y., Iwamoto, M., Shimono, K. \& Kamo, N. Association of pharaonis phoborhodopsin with its cognate transducer decreases the photo-dependent reactivity by water-soluble reagents of azide and hydroxylamine. Biochim. Biophys. Acta 1558, 63-69 (2002).

29. Sudo, Y., Iwamoto, M., Shimono, K. \& Kamo, N. Tyr-199 and charged residues of pharaonis phoborhodopsin are important for the interaction with its transducer. Biophys. $J$. 83, 427-432 (2002).

30. Yang, C. S. \& Spudich, J. L. Light-induced structural changes occur in the transmembrane helices of the Natronobacterium pharaonis HtrII transducer. Biochemistry 40, 14207-14214 (2001). 
31. Gordeliy, V.I., Labahn, J., Moukhametzianov, R., Efremov, R., Granzin, J., Schlesinger, R., Büldt, G., Savopol, T., Scheidig, A. J., Klare, J. P. \& Engelhard, M. Molecular basis of transmembrane signalling by sensory rhodopsin II- transducer complex. Nature 419, 484-487 (2002).

32. Hirayama, J., Imamoto, Y., Shichida, Y., Kamo, N., Tomioka, H. \& Yoshizawa, T. Photocycle of phoborhodopsin from haloalkaliphilic bacterium (Natronobacterium pharaonis) studied by low-temperature spectrophotometry. Biochemistry 31, 2093-2098 (1992).
33. Anderson, J.L., Frase, H., Michaelis, S. \& Hrycyna, C. A. Purification, functional reconstitution, and characterization of the Saccharomyces cerevisiae isoprenylcysteine carboxylmethyltransferase Ste14p. J. Biol. Chem. 280, 7336-7345 (2005).

34. Lacapere, J. J., Robert, J. C. \& Thomas-Soumarmon, A. Efficient solubilization and purification of the gastric $\mathrm{H}^{+}, \mathrm{K}^{+}$ ATPase for functional and structural studies. Biochem. J. 345, 239-245 (2000). 